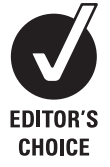

- Additional figures are published online only at http:// ard.bmj.com/content/vol68/ issue 10

${ }^{1}$ Regeneron Pharmaceuticals, Tarrytown, New York, USA;

${ }^{2}$ Queens College and The Graduate Center of the City University of New York, New York, New York, USA; ${ }^{3}$ VA Medical Center and the University of California at San Diego, La Jolla, California

Correspondence to:

Dr R Torres, Regeneron

Pharmaceuticals, 777 Old Saw

Mill River Rd, Tarrytown, NY

10591, USA; richard.torres@ regeneron.com

Accepted 19 May 2009 Published Online First

14 June 2009

\title{
Hyperalgesia, synovitis and multiple biomarkers of inflammation are suppressed by interleukin 1 inhibition in a novel animal model of gouty arthritis
}

\author{
R Torres, ${ }^{1}$ L Macdonald, ${ }^{1}$ S D Croll, ${ }^{1,2}$ J Reinhardt, ${ }^{1}$ A Dore, ${ }^{1}$ S Stevens,, D M Hylton, \\ J S Rudge, ${ }^{1}$ R Liu-Bryan, ${ }^{3}$ R A Terkeltaub, ${ }^{3}$ G D Yancopoulos, ${ }^{1}$ A J Murphy ${ }^{1}$
}

\begin{abstract}
Background: Monosodium urate (MSU) and calcium pyrophosphate dihydrate (CPPD) crystal-induced interleukin $1 \beta$ (IL1 $\beta$ ) release contributes to inflammation in subcutaneous air pouch and peritoneal models of acute gout and pseudogout. However, consequences of IL1 inhibition have not been explored in more clinically relevant models of crystal-induced arthritis.

Objective: To develop a novel mouse model of acute gouty ankle arthritis and use it to assess the effects of genetic deletion of IL1 receptor type (IL1R1) and of exogenous mIL1 Trap (a high-affinity blocker of mouse $\mathrm{IL} 1 \alpha$ and IL1 $\beta)$ on pain, synovitis and systemic inflammatory biomarkers.
\end{abstract}

Methods: MSU crystals were injected into the mouse ankle joint and pain and ankle swelling were measured over 4 days. The effects of IL1 inhibition were determined in this model, and in the comparator models of crystalinduced peritonitis and subcutaneous air pouch inflammation.

Results: Both IL1R1-null mice and mice pretreated with mIL1 Trap showed reduced neutrophil influx in MSU and CPPD crystal-induced peritonitis and air pouch models $(p<0.05)$. In the ankle joint model, both IL1R1 knockout mice and pretreatment with mIL1 Trap were associated with significant reductions in MSU crystal-induced elevations in hyperalgesia, inflammation, serum amyloid $\mathrm{A}$ and the levels of multiple inflammatory cytokines and chemokines $(p<0.05)$. Additionally, it was found that administration of mIL1 Trap after MSU crystal injection reduced established hyperalgesia and ankle swelling. Conclusions: IL1 inhibition both prevented and relieved pain and ankle joint inflammation in response to intraarticular MSU crystals in mice. Results suggested that IL1 Trap has the potential to both prevent and treat gouty arthritis.

Acute gouty arthritis, an extremely painful condition, ${ }^{1}$ is provoked by activation of multiple inflammatory mediators by uric acid crystals. ${ }^{2}$ This process is driven by neutrophil influx into the joint and is associated with systemic manifestations such as fever, leukocytosis and elevated acute phase reactants. ${ }^{3-5}$

Monosodium urate (MSU) crystal-induced release of interleukin $1 \beta$ (IL1 $\beta),{ }^{67}$ mediated by NLRP3 inflammasome activation, ${ }^{8}$ is central to experimental gouty inflammation. ${ }^{9}$ The NLRP3 inflammasome acts as an activating scaffold for caspase-1, which cleaves and activates pro-IL1 $\beta$ into mature IL1 $\beta .{ }^{10}$ Macrophages from mice deficient in inflammasome components cryopyrin,
ASC, or caspase-1, or in the IL1 receptor IL1R1, have reduced neutrophil influx in response to MSU crystals in vivo.9 ${ }^{11}$ Additionally, pharmacological blockade of the IL1 pathway reduces neutrophil influx in response to MSU using either recombinant IL1 receptor antagonist (IL1ra; anakinra) or blocking antibodies to IL1 and IL1R1. ${ }^{11} 12$

Consequences of IL1 blockade have not been explored in the joint in gout animal models. In this study we developed a mouse model of gout-like joint hyperalgesia and inflammation in response to MSU crystals in the ankle. Systemic IL1 inhibition, using IL1R1 knockouts or mIL1 Trap, prevented and suppressed ankle hyperalgesia and synovitis.

\section{METHODS}

\section{Subjects}

Male IL1R1 knockouts on a C57BL/6 background and $\mathrm{C} 57 \mathrm{BL} / 6$ age-matched control mice were obtained from the Jackson Laboratory (stock no 003245; Bar Harbor, Maine, USA) and used between the ages of 12 and 16 weeks. For mIL1 Trap and colchicine studies, male C57BL/ 6 mice were obtained from the Jackson Laboratory or Taconic Laboratories (Hudson, New York, USA). Mice were individually housed for at least 1 week before study on a standard 12:12 h light-dark cycle (lights on 07:00). Food and water were freely available. All experiments were approved by the Regeneron Institutional Animal Care and Use Committee.

\section{Reagents}

MSU crystals were prepared as described previously. ${ }^{13}$ Monoclinic calcium pyrophosphate dihydrate (CPPD) crystals were kindly provided by $\mathrm{Dr}$ Kenneth Pritzker and incubated for $2 \mathrm{~h}$ at $200^{\circ} \mathrm{C}$. Both MSU and CPPD crystals were suspended at $25 \mathrm{mg} / \mathrm{ml}$ in sterile endotoxin-free phosphate-buffered saline (PBS; Gibco, Carlsbad, California, USA) and verified to be free of detectable lipopolysaccharide contamination by Limulus amebocyte lysate assay (Cambrex, East Rutherford, New Jersey, USA).

Murine IL1 Trap (5-35 mg/kg) was used for pharmacological IL1 inhibition because the high affinity human IL1 Trap (rilonacept) cross-reacts poorly with rodent IL1 $\beta .{ }^{14}$ mIL1 Trap was dissolved in $10 \mathrm{mM}$ phosphate, $100 \mathrm{mM} \mathrm{NaCl}, 0.004 \%$ polysorbate 20, $\mathrm{pH}$ 6.5. Mouse Fc control protein was dissolved in $10 \mathrm{mM}$ Tris, $150 \mathrm{mM} \mathrm{NaCl}, 5 \%$ glycerol, $\mathrm{pH}$ 7.5. Colchicine (Sigma-Aldrich, St Louis, Missouri, USA) was suspended in saline at $1 \mathrm{mg} / \mathrm{ml}$ and diluted into working solutions. 


\section{Crystal-induced peritonitis and subcutaneous (SC) air pouch models}

Mice were intraperitoneally injected with $1 \mathrm{mg} \mathrm{MSU}$ or CPPD crystals resuspended in $0.5 \mathrm{ml}$ endotoxin-free PBS (Cambrex, East Rutherford, New Jersey, USA). After 6 h, mice were killed and their peritoneal cavities washed and harvested with $10 \mathrm{ml}$ stain buffer (BD Pharmingen, San Jose, California, USA). The air pouch model was developed as previously described..$^{13}$ Six hours after crystal injection, air pouches of these mice were harvested with $5 \mathrm{ml}$ stain buffer. Isolated cells were incubated with the monoclonal antibody CD16/CD32 (mouse BD Fc Block, BD Pharmingen) to block Fc $\gamma$ RII/III receptors. Cells were then stained with a neutrophil-specific monoclonal antibody (PE-Ly6G (R-phycoerythrin (R-PE)-conjugated rat anti-mouse Ly-6G monoclonal antibody); BD Pharmingen) and sorted (MoFlo high-speed cell sorter; Beckman Coulter, Fullerton, California, USA). The total number of lavage neutrophils was determined by dividing the number of cells counted by the percentage lavage recovery. This number was multiplied by the percentage Ly-6G-positive cells from the FACS count.

\section{Crystal-induced ankle arthritis model and behavioural testing}

MSU crystals $(0.5 \mathrm{mg}$ ) suspended in $20 \mu$ l endotoxin-free PBS or PBS control were injected intra-articularly into the tibio-tarsal joint (ankle) of mice anaesthetised with $2.5 \%$ isoflurane. Mice were assessed for nociceptive threshold and inflammation for up to 4 days. Before behavioural testing, mice were acclimatised to the testing room for at least $1 \mathrm{~h}$. All behavioural measures were taken by experimenters blind to genotype or treatment. Baseline readings were obtained for each measure before injections.
To study thermal hyperalgesia after ankle injections, the Hargreaves' test was performed as previously described. ${ }^{15}$ Briefly, a radiant heat source was applied to the heel and the latency for the animal to withdraw its foot was recorded. For each mouse, three readings were obtained and the median latency was used. The results are presented as the percentage change from the baseline readings.

To test willingness to bear weight on injected ankles, the weightbearing test was performed using an Incapacitance meter (model 600, IITC Life Sciences, Woodland Hills, California, USA). Each hind paw was placed on a transducer pad and the weight distribution, in grams, was recorded over $4 \mathrm{~s}$. The results are presented as percentage weight distributed to the affected limb.

The diameter of ankle joints was measured with callipers while mice were anaesthetised under $2.5 \%$ isoflurane. The results are presented as percentage change from baseline diameter.

For histological analyses, at least 10 mice per group were killed 1 day after ankle injection. The ankle region was isolated and fixed in buffered neutral 10\% formalin (VWR, West Chester, Pennsylvania, USA), embedded in paraffin and sectioned at $5 \mu \mathrm{m}$ for staining with haematoxylin and eosin (Histoserv, Germantown, Maryland, USA).

\section{Mouse serum amyloid A (SAA) and cytokine profiling}

Mice were killed one day after ankle injection and blood was collected by cardiac puncture. Whole blood $(100 \mu \mathrm{l})$ was transferred to a serum separator tube (Microtainer Brand SST, Becton Dickinson, Franklin Lanes, New Jersey, USA) and SAA
Figure 1 Blocking interleukin 1 (IL1) reduced neutrophil influx in crystalinduced peritonitis and air pouch models. (A) IL1-R1 knockouts (KOs) show fewer monosodium urate (MSU) crystal-induced peritoneal neutrophils (phosphatebuffered saline (PBS): $n=6$ wild-type (WT); $n=7$ IL1R1 K0/MSU: $n=11$ WT; $\mathrm{n}=10$ IL1R1 KO). (B) mlL1 Trap dosedependently inhibited MSU-induced peritoneal neutrophils (PBS: $n=4$ vehicle; $\mathrm{n}=4,35 \mathrm{mg} / \mathrm{kg}$ mlL1 Trap/MSU: $\mathrm{n}=5,12.5 \mathrm{mg} / \mathrm{kg} \mathrm{mFc;} \mathrm{n}=6,5 \mathrm{mg} / \mathrm{kg}$ mlL1 Trap; $n=6,15$ mg/kg mlL1 Trap; $\mathrm{n}=5,35 \mathrm{mg} / \mathrm{kg}$ IL1 Trap). (C) IL1R1 KOs showed fewer MSU and calcium pyrophosphate dihydrate (CPPD) crystalinduced air pouch neutrophils (PBS: $n=6$ WT; $n=5$ IL1R1 KO/crystals: $n=6$ each). (D) mIL1 Trap dose-dependently decreased MSU-induced air pouch neutrophils. mIL1 Trap decreased CPPD crystal-induced air pouch neutrophils (PBS: $n=5$ vehicle/MSU: $n=6$ vehicle; $\mathrm{n}=5,5 \mathrm{mg} / \mathrm{kg} \mathrm{mlL} 1 \mathrm{Trap} ; \mathrm{n}=5,15 \mathrm{mg} /$ $\mathrm{kg}$ mlL1 Trap; $\mathrm{n}=5,35 \mathrm{mg} / \mathrm{kg} \mathrm{mlL} 1$ Trap/CPPD: $n=5$ each). \# $p<0.001$ compared with controls. Data expressed as means (SEM).
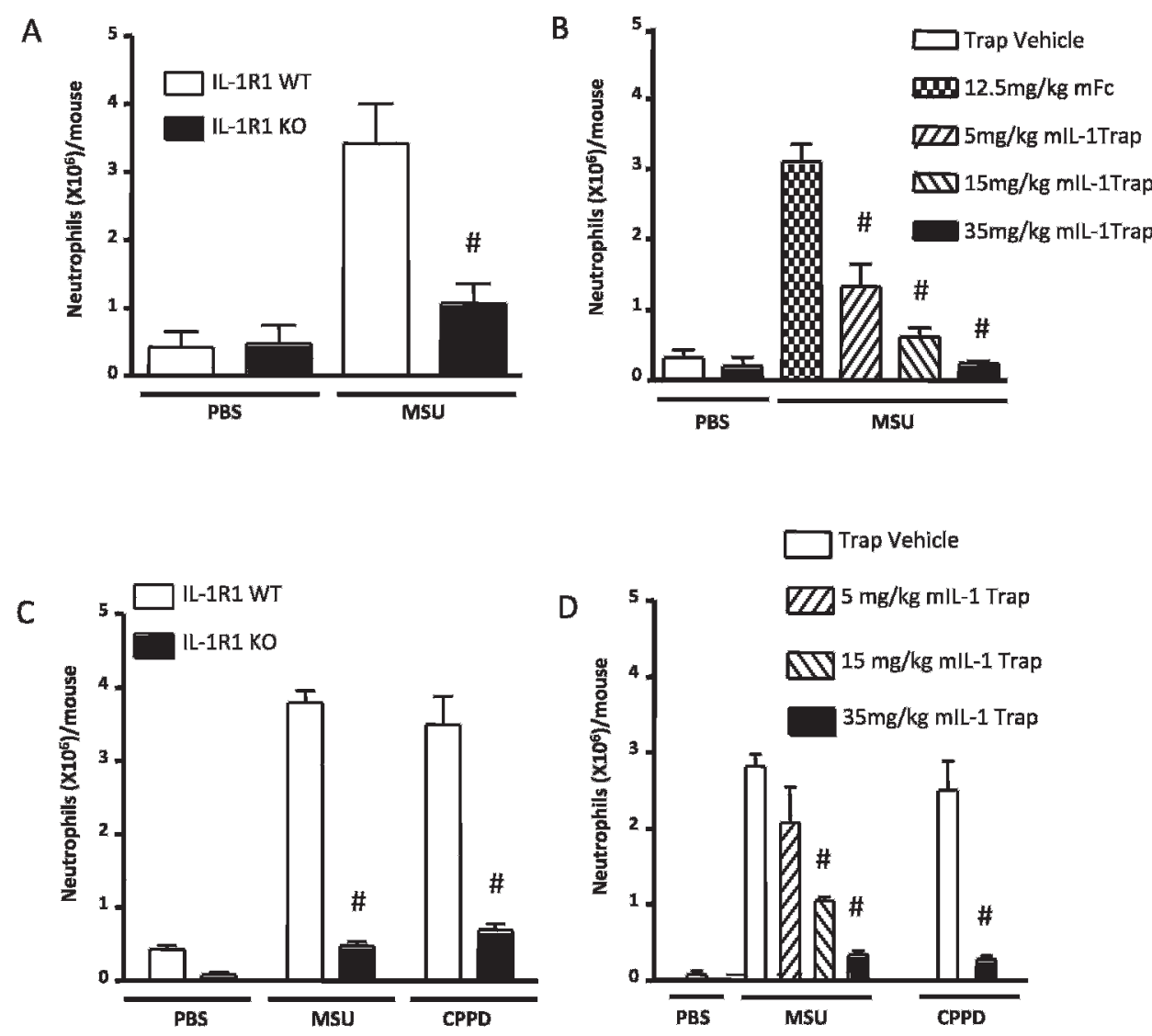
Figure 2 Blockade of the interleukin 1 receptor 1 (IL1R1) pathway reduced thermal hyperalgesia and weightbearing asymmetry in an in vivo model of acute gout. (A) After intra-articular injection of $0.5 \mathrm{mg}$ monosodium urate (MSU) crystals into the ankle, thermal hyperalgesia in the Hargreaves' test, occurred in wild-type mice (WTs); IL1R1 knockout mice (KOs) had significantly reduced hyperalgesia (phosphate-buffered saline (PBS): $n=5$ per group; MSU: $\mathrm{n}=6$ per group). (B) The effects of MSU crystal ankle injection on weightbearing distribution of the hind paws. IL1R1 KOs showed decreased weightbearing asymmetry on the injured hind paw compared with WTs (PBS: $n=5$ per group/MSU: $n=6$ per group). mIL1 Trap (35 mg/kg) prevented the nociceptive responses detected in the Hargreaves' test (C) and in the weightbearing test (D) in the same model (PBS: $n=5$ per group/ MSU: $n=9$ per group). No effects of PBS ankle injections were detected in any of the behavioural tests (A-D). ${ }^{*} p<0.05$ compared with WT or vehicle-treated mice. Data are expressed as means (SEM).
A

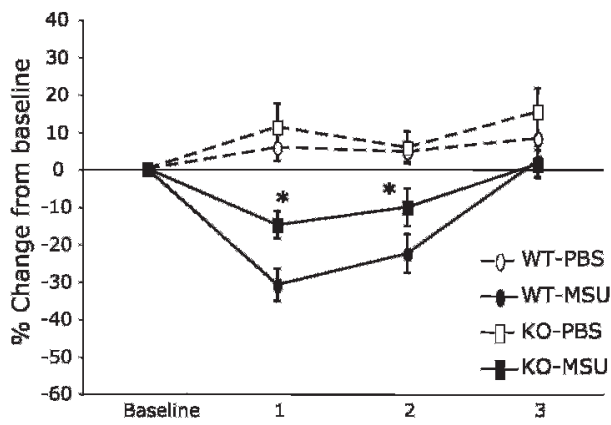

Days after MSU Crystals Ankle Injection

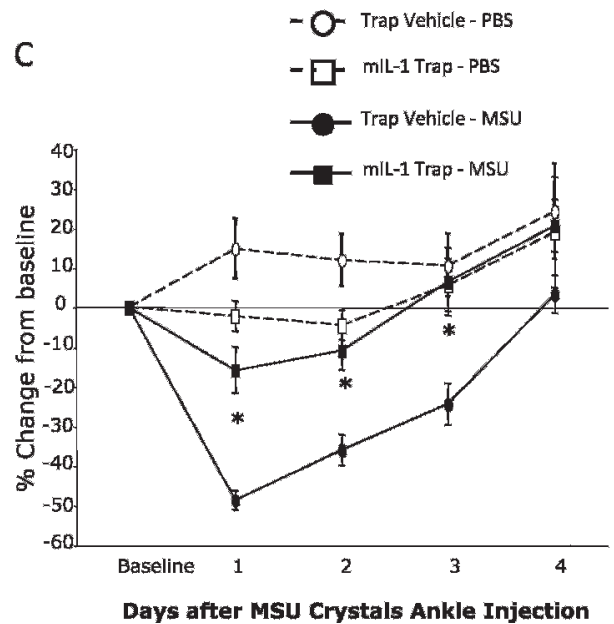

B

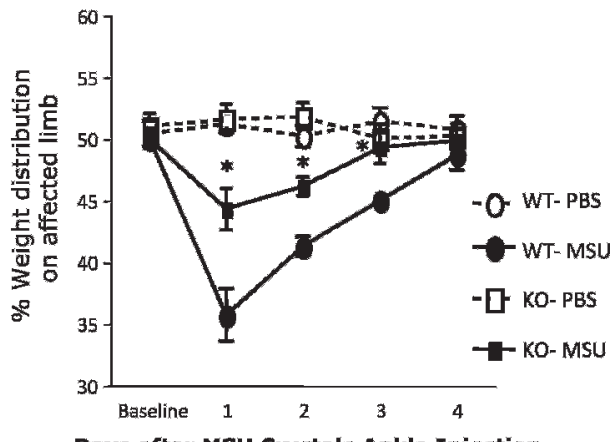

Days after MSU Crystals Ankle Injection

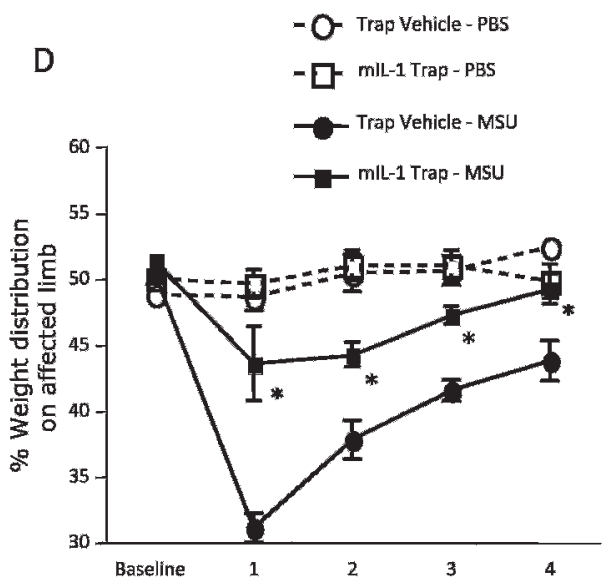

Days after MSU Crystals Ankle Injection
Figure 3 Inflammatory responses were attenuated by interleukin 1 (IL1) signalling deficiency in an in vivo acute gout model. (A) IL1R1 knockout mice (KOs) showed decreased ankle diameter after monosodium urate (MSU) injection compared with wild-type mice (WTs) (phosphate-buffered saline (PBS): $n=5$ per group/MSU: $n=6$ per group). (B) mlL1 Trap (35 mg/kg) significantly decreased ankle diameter (PBS: $n=5$ per group/MSU: $n=9$ per group).

Photomicrographs of median H\&E-stained ankles showed that IL1R1 KOs show less cell recruitment to MSU crystals than WTs (C), as do mIL1 Trap-treated mice compared with vehicle-treated mice (D). Arrows indicate ankle infiltrate. Blood serum amyloid $A$ (SAA) was measured 1 day after ankle injection. (E) SAA levels were reduced in IL1R1 $\mathrm{KO}$ s relative to WTs (PBS: $n=8$ per group/MSU: $n=10$ per group). (F) mlL1 Trap prevented increased SAA (PBS: $n=6$ vehicle; $\mathrm{n}=10$ mlL1 Trap; MSU: $\mathrm{n}=14$ vehicle; $\mathrm{n}=12$ mlL1 Trap). ${ }^{*} \mathrm{p}<0.05,+\mathrm{p}<0.01$, $\# p<0.001$ compared with WTs or vehicle controls. Results expressed as means (SEM).
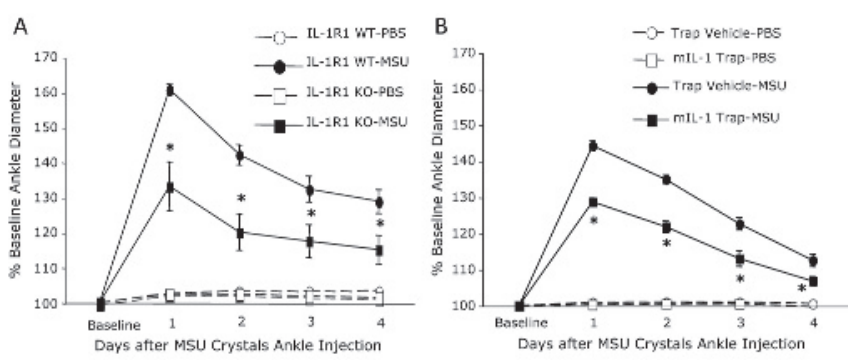

C
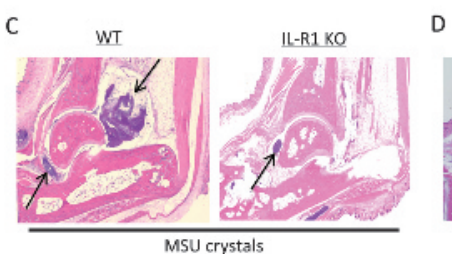

Vehicle
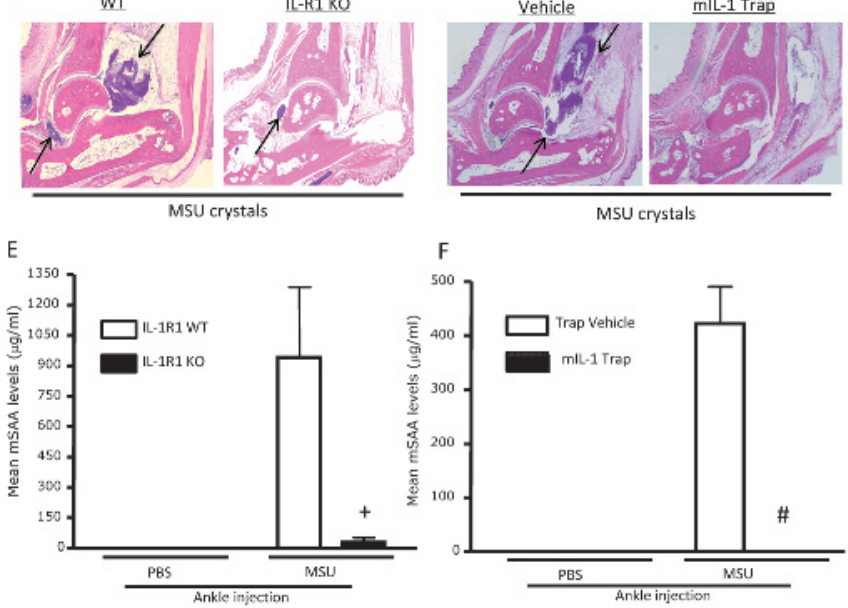
Figure 4 Murine interleukin 1 (mlL1) Trap was as efficacious as a high dose of colchicine against neutrophil influx, pain and swelling in in vivo gout models. No synergism was detected for mlL1 Trap and high-dose colchicine combination therapy. (A) A dose-dependent decrease in neutrophil influx was achieved with increasing doses of colchicine pretreatment in the monosodium urate (MSU) crystal-induced peritonitis model (phosphate-buffered saline (PBS): $n=6$ per group/MSU: $n=6$ per group). (B) In the same model, mlL1 Trap $(35 \mathrm{mg} / \mathrm{kg}$ ) pretreated mice showed a reduction in the number of neutrophils equivalent to the high dose of colchicine $(2 \mathrm{mg} / \mathrm{kg})$ (PBS: $n=3$, saline/MSU: $n=2$, saline; $\mathrm{n}=6$, Trap vehicle; $\mathrm{n}=6,35 \mathrm{mg} / \mathrm{kg}$ $\mathrm{mlL1}$ Trap; $\mathrm{n}=6,1 \mathrm{mg} / \mathrm{kg}$ colchicine; $\mathrm{n}=6,2 \mathrm{mg} / \mathrm{kg}$ colchicine). (C and D) Mice were treated with mlL1 Trap, colchicine, or a combination of the two drugs before injection of MSU crystals into the ankles. (C) Thermal hyperalgesia in the Hargreaves' test was significantly reduced by mlL1 Trap. (D) Ankle swelling was significantly reduced by mlL1 Trap or colchicine. ${ }^{*} p<0.05,+p<0.01$, $\# \mathrm{p}<0.001$ compared with saline- or vehicle-treated mice. Results are expressed as means (SEM).
A

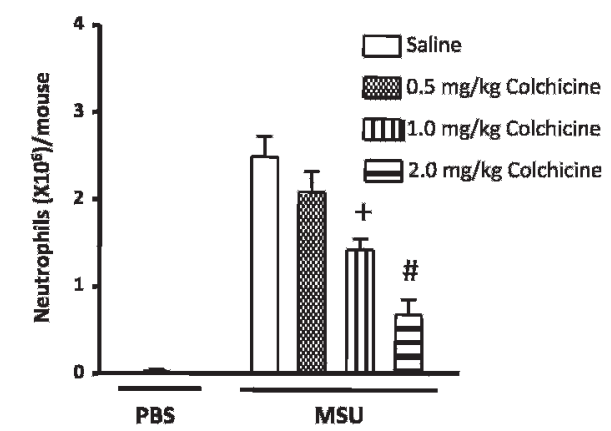

C
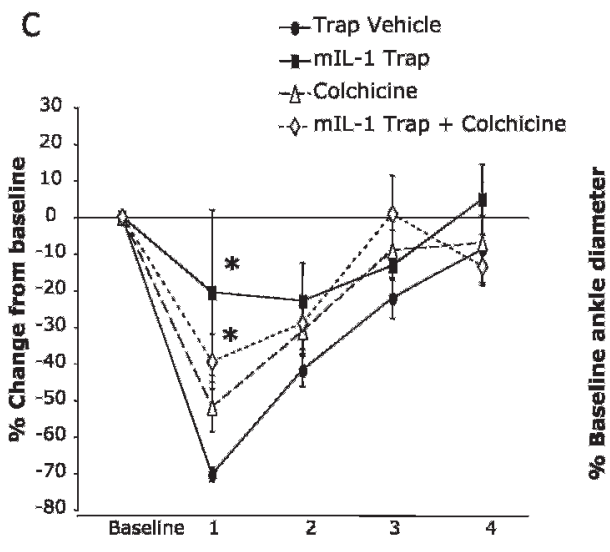

Days after MSU Crystals Ankle Injection
B
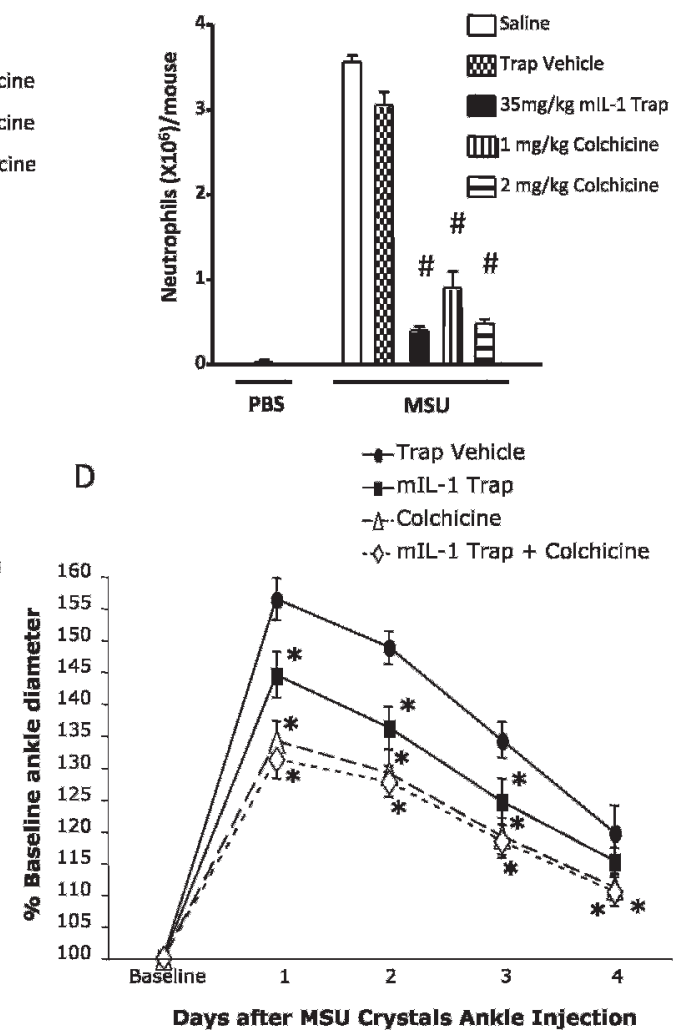

levels were measured using a mouse SAA ELISA kit (Biosource, Carlsbad, California, USA), according to manufacturer's instructions. Injected ankles were dissected and homogenised in a protease inhibitor cocktail (1 tablet/50 ml, Boehringer Manheim, Indianapolis, Indiana, USA) using a Mixer Mill MM 300 (Qiagen, Valencia, California, USA). Homogenates were centrifuged and supernatants analysed according to the manufacturer's instructions with a Luminex instrument (Luminex, Austin, Texas, USA) and a mouse cytokine/ chemokine multiplex panel that detected 22 different proteins (mIL1 $\alpha$, mIL1 $\beta$, mIL2, mIL4, mIL5, mIL6, mIL7, mIL9, mIL10, mIL12 (p70), mIL13, mIL15, mIL17, mIFN $\gamma$, mIP10, mG-CSF, mGM-CSF, mTNF $\alpha$, mKC, mMCP1, mMIP- $1 \alpha$ and mRantes) (Millipore, Billerica, Massachusetts, USA).

\section{Statistical analyses}

Data are expressed as means (SEM) and were analysed using analysis of variance (ANOVA) and post hoc tests to probe significant effects (Bonferroni tests for one- or two-way ANOVAs; Tukey HSD tests for three-way ANOVAs).

\section{RESULTS}

IL1 signalling mediates crystal-induced inflammation in the peritoneum and synovium-like air pouch

We confirmed ${ }^{11}$ a marked decrease in neutrophil ingress in the IL1R1 knockout (KO) mice compared with wild-type (WT) mice in the MSU-induced peritonitis model (fig 1A). Next, prophylactic subcutaneous (SC) injections of mIL1 Trap were administered on alternate days for three treatments, with the last given the day before MSU crystal injections. mIL1 Trap produced a significant dose-dependent decrease in neutrophil ingress (fig 1B). Similar decreases were seen using the CPPD crystal-induced peritonitis model of pseudogout ${ }^{9}$ (online supplementary figure 1). In contrast, intraperitoneal injection of $0.2 \mathrm{mg}$ zymosan caused comparable neutrophil influx in IL1 R1 KOs and WTs (data not shown). Hence, IL1R1 KOs did not exhibit a general deficiency in neutrophil recruitment.

To investigate the effects of IL1 inhibition on MSU and CPPD crystal-induced inflammation in a synovitis model, we used SC air pouches. ${ }^{13}{ }^{16}$ In response to either MSU or CPPD, significant reductions in neutrophil influx were detected in IL1R1 KOs compared with WTs (fig 1C) and in mIL1 Trap-treated versus vehicle-treated mice (fig 1D).

\section{IL1 inhibition prevented hyperalgesia in a murine model of acute gouty arthritis}

Animals exhibited thermal hyperalgesia for 2 days after an intra-articular ankle injection of MSU crystals, peaking 1 day after injection (fig 2A). This thermal hyperalgesia was significantly reduced in IL1R1 KOs compared with WTs. No hyperalgesia was detected in mice of either genotype with ankle injection of PBS (fig 2A).

Ankle injections of MSU, but not PBS, induced a significant shift in weight bearing away from the affected limb in WTs, peaking one day after injection (fig 2B). In contrast, IL1R1 KOs showed significantly less weight redistribution. In the same model, mIL1 Trap treatment significantly reduced both thermal hyperalgesia in the Hargreaves' test (fig 2C) and weight redistribution in the weightbearing test (fig $2 \mathrm{D}$ ).

Inhibition of IL1 reduced inflammation in a murine model of acute gout

After injections of $\mathrm{MSU}$, mice developed ankle swelling that peaked after 1 day (figs 3A and B). Both IL1R1 KOs (fig 3A) and 
A
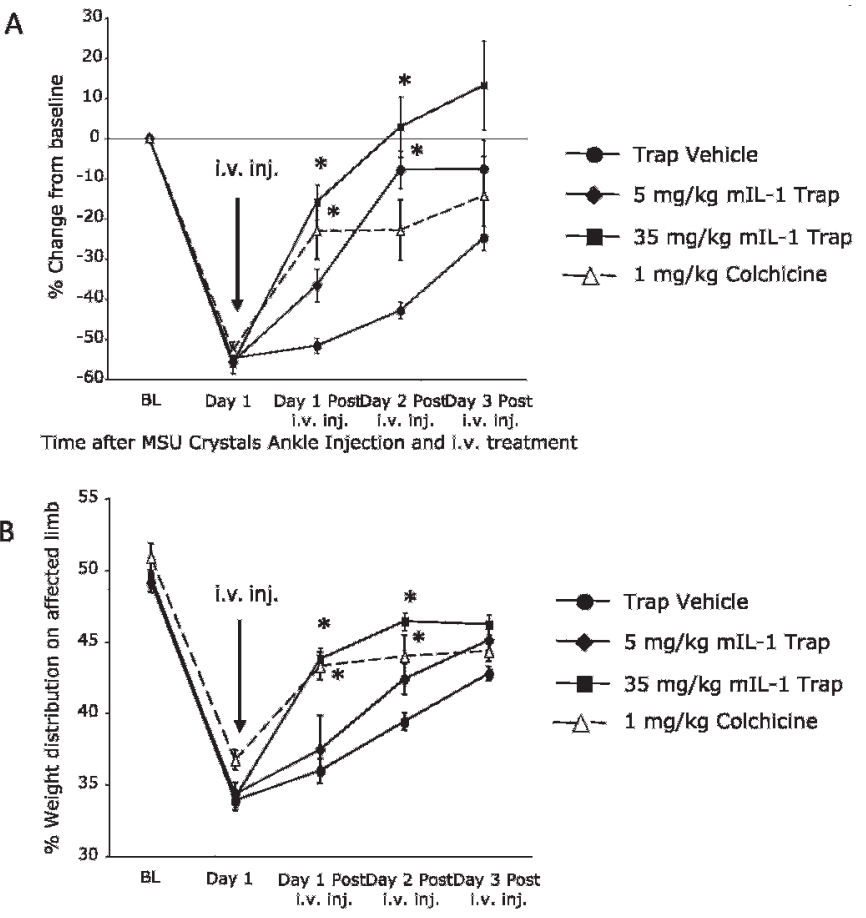

Time after MSU Crystals Ankle Injection and i.v. treatment

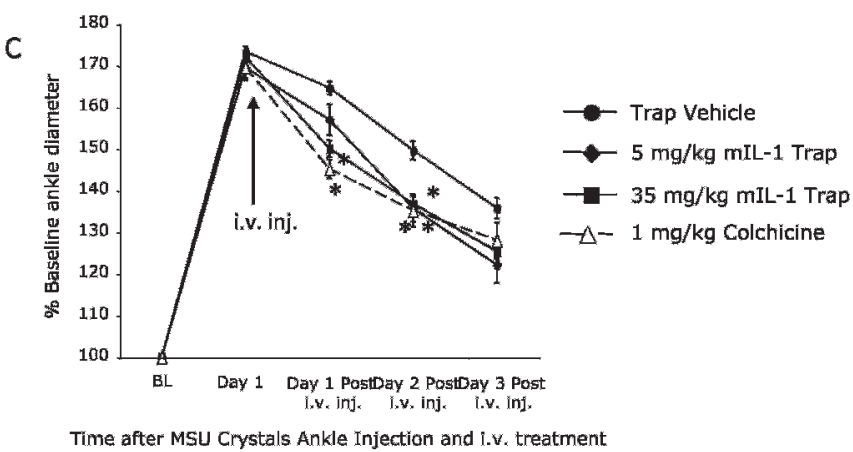

Figure 5 Murine interleukin 1 (mIL1) Trap was as efficacious as highdose colchicine in relieving established pain and swelling when given 1 day after ankle injection of monosodium urate (MSU) crystals.

(A) mlL1 Trap and colchicine treatments significantly reduced thermal hyperalgesia for 3 days after treatment. (B) Mice treated with both mlL1 Trap and colchicine showed a significant decrease in the weightbearing asymmetry. (C) Ankle diameter studies showed that mice treated with mlL1 Trap and colchicine experienced similar reductions in ankle diameter ( $n=14$, Trap vehicle; $n=16$, mlL1 Trap; $n=15$, colchicine for A-C) ${ }^{*} p<0.05$ compared with vehicle treated mice. Results are expressed as means (SEM).

mice treated with mIL1 Trap (fig 3B) showed significantly less swelling.

Control injections of PBS into the ankle did not cause inflammation as assessed histologically in any of the groups (data not shown). Both WTs (fig 3C) and mice injected with vehicle (fig $3 \mathrm{D}$ ) exhibited a dramatic cellular infiltrate in the ankle after MSU injection. In contrast, IL1R1 KOs (fig 3C) and mice treated with mIL1 Trap (fig 3D) showed marked reduction in this infiltrate.

The acute phase protein SAA rises during acute gout flares in patients and subsequently decreases with treatment. ${ }^{17} \mathrm{MSU}$ crystal injections significantly increased SAA (figs $3 \mathrm{E}$ and $\mathrm{F}$ ). IL1R1 KOs developed less SAA elevation than WTs (fig 3E) and mIL1 Trap blunted SAA induction (fig 3F).
mIL1 Trap is as effective as high doses of colchicine in preventing pain and inflammation

Colchicine dose-dependently decreased neutrophil influx in crystal-induced peritonitis (fig 4A). mIL1Trap was as effective as the two highest doses of colchicine in preventing influx in both MSU- and CPPD crystal-induced peritonitis (fig 4B; data not shown). To test colchicine in the ankle model, we injected $1 \mathrm{mg} / \mathrm{kg}$ colchicine SC concurrent with the ankle injection and then administered an additional $0.5 \mathrm{mg} / \mathrm{kg}$ on days $1-4$ after MSU. This dosing schedule was the highest colchicine regimen tolerated without causing weight loss and lethargy (data not shown). Mice were treated with mIL1 Trap, colchicine, or both and then injected with MSU crystals into the ankle. Thermal hyperalgesia was significantly reduced by mIL1 Trap, but not colchicine, and no synergistic effect was detected with cotreatment (fig 4C). Ankle swelling was decreased by both mIL1 Trap and colchicine in the first 3 days after ankle injection; the combination therapy did not lead to any greater reduction (fig 4D).

\section{mIL1 Trap provides relief of established pain and inflammation similar to colchicine}

To study efficacy in relieving established pain and inflammation, mice were injected with a single tail vein intravenous injection of mIL1 Trap (5 or $35 \mathrm{mg} / \mathrm{kg}$ ) or colchicine $(1 \mathrm{mg} / \mathrm{kg}$ ) 1 day after ankle injection of MSU. Significant relief of thermal hyperalgesia was achieved with both doses of mIL1 Trap and with colchicine (fig $5 \mathrm{~A}$ ). In the weightbearing test, a significant reduction of weightbearing redistribution was seen in mice treated with $35 \mathrm{mg} / \mathrm{kg}$ mIL1 Trap or colchicine (fig 5B). Both mIL1 Trap and colchicine significantly decreased ankle swelling (fig 5C). Therefore, mIL1 Trap reduced hyperalgesia and inflammation as effectively as colchicine even when administered after the pain and inflammation were established.

\section{IL1 inhibition attenuates cytokine and chemokine upregulation in the ankle model}

To identify cytokines and chemokines altered by ankle injection of MSU crystals, as well as to study the role of IL1 in their regulation, we performed cytokine and chemokine profiling on ankles and serum samples collected $24 \mathrm{~h}$ after ankle injection. Luminex profiling confirmed increased IL1 $\beta$ in MSU crystalinjected ankles (table 1). Additionally, six other cytokines, IL6, monocyte chemotactic protein-1 (MCP-1; CCL2), macrophage inhibitory protein-1 (MIP-1 $\alpha$; CCL3), KC (CXCL1), tumour necrosis factor $\alpha(\mathrm{TNF} \alpha)$ and granulocyte-colony stimulating factor (G-CSF) were upregulated in the injected ankles and three others, IL6, KC and G-CSF, in the serum. In contrast, IL1 $\alpha$ was significantly decreased with MSU treatment (online supplementary fig 2). A significant attenuation of MSU-induced increases in cytokines and chemokines was seen with genetic deletion of IL1R1 and treatment with mIL1 Trap (table 1), though normalisation of IL1 $\alpha$ was not observed.

\section{DISCUSSION}

This study reinforced the hypothesis that IL1 signalling plays a critical role in MSU and CPPD crystal-induced inflammation, ${ }^{911}{ }^{12}$ including joint inflammation. ${ }^{23}{ }^{18}{ }^{19}$ Furthermore, we described a novel mouse model of crystal-induced synovitis and associated hyperalgesia. As previously reported in rat studies of intra-articular injections of MSU crystals, ${ }^{20}{ }^{21}$ we observed a reluctance to place full weight on the injected hind leg and ankle swelling. Additionally, as observed in rats, ${ }^{20} 21$ hyperalgesic 
Table 1 Luminex data for mean cytokine and chemokine levels (pg/ml) in ankles (regular font) and serum (italicised font) for studies of genetic (IL1R1 knockout mice) or pharmacological (mIL1 Trap) depletion of IL1 signalling

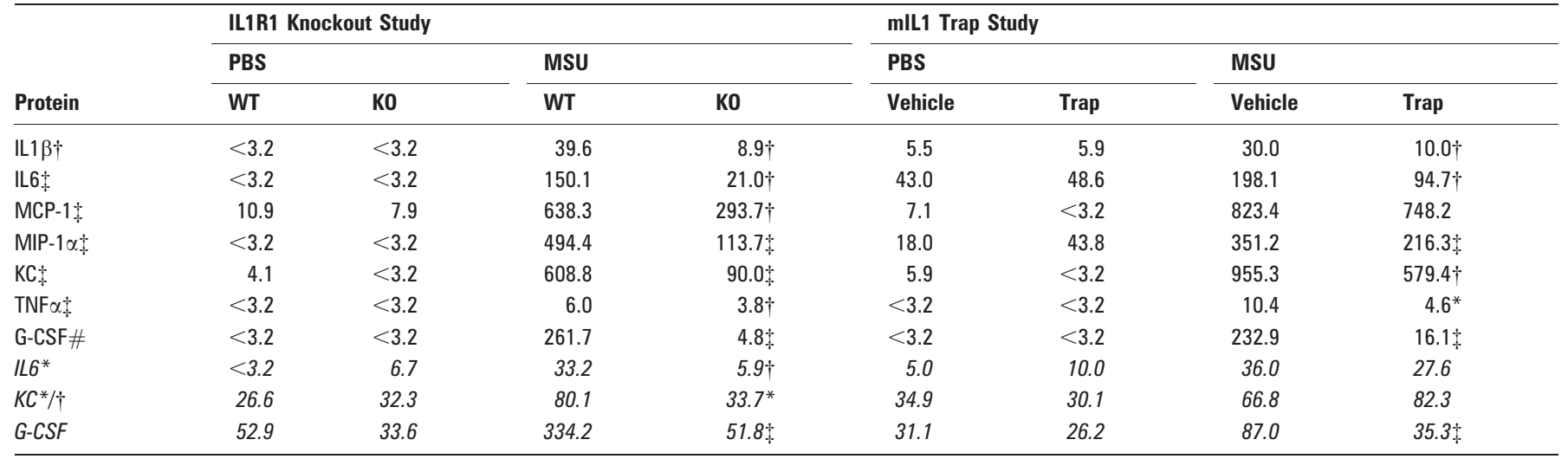

Only proteins significantly upregulated by MSU crystals are shown (level of significance indicated after protein name). Asterisks after data values indicate that the value is significantly different from controls (WT or vehicle), ${ }^{*} p<0.05, \dagger p<0.01, \pm p<0.001$.

G-CSF, granulocyte-colony stimulating factor; IL1R1, interleukin 1 receptor type 1; KC, keratinocyte chemoattractant; K0, knockout; MCP-1, monocyte chemotactic protein-1; mIL1, murine interleukin 1; MIP-1, macrophage inhibitory protein-1; MSU, monosodium urate; PBS, phosphate-buffered saline; TNF, tumour necrosis factor; WT, wild-type.

responses were fully developed within 1 day and subsided after several days.

mIL1 Trap was generally as effective as genetic deletion of IL1R1 against both inflammation and hyperalgesia in our model. Colchicine was previously established to inhibit MSUinduced neutrophil influx in rats, ${ }^{22}{ }^{23}$ and here we showed that mIL1 Trap worked as well as 1 or $2 \mathrm{mg} / \mathrm{kg}$ of colchicine. However, these colchicine doses are near the mouse $\mathrm{LD}_{50}$ of $3 \mathrm{mg} / \mathrm{kg}$ intraperitoneally ${ }^{22}$ and identical to the $2 \mathrm{mg} / \mathrm{kg}$ dose which reduced oedema by $71 \%$ but resulted in the death of $75 \%$ of the animals in a rat study. ${ }^{23}$ Our finding that both mIL1 Trap and maximum tolerated doses of colchicine relieve MSU crystalinduced hyperalgesia does not necessarily indicate a commonality of mechanism. High-dose colchicine does, however, suppress IL1 $\beta$ maturation and release. ${ }^{9}$

Luminex-based cytokine assays revealed upregulation of multiple cytokine mediators of gouty inflammation, including IL1 $\beta$, at the injection site. ${ }^{18-29}$ Our observation that MSU injection decreased IL $1 \alpha$ was unexpected and strengthens the argument that it is IL1 $\beta$ which mediates MSU-induced pain and inflammation. Furthermore, it suggests that it is inhibition of IL1 $\beta$, not IL1 $\alpha$, which underlies the therapeutic effects of mIL1 Trap and genetic deletion of IL1R1. IL1 inhibition caused significant reduction in multiple inflammatory cytokines, including IL6, MCP-1 and KC, ${ }^{112425} 30$ but did not completely normalise the levels of these cytokines, suggesting contributions from IL1-independent pathways. IL1R1 KOs showed more attenuation than mice treated with mIL1 Trap, suggesting potentially better inhibition with genetic deletion of the IL1 receptor than with pharmacological inhibition of IL1.

IL1 modulates other pain mediators such as nerve growth factor, prostaglandin, substance-P and matrix metalloproteinase- $9,{ }^{26}$ and also modulates neuronal excitability directly via TRPV1, sodium channels, $\gamma$-aminobutyric acid (GABA) receptors and $\mathrm{N}$-methyl D-aspartate (NMDA) receptors. ${ }^{27}$ The beneficial effect of IL1 $\beta$ inhibition on gout-related pain could therefore operate via a number of candidate mechanisms. Our newly characterised mouse model could serve as a platform to assess the relative contributions of these pathways.

In summary, IL1 $\beta$ drives both hyperalgesia and inflammation in a mouse model of gout, and mIL1 Trap both prevents and treats crystal-induced hyperalgesia and inflammation. The longacting IL1 blocker rilonacept, the human version of IL1 Trap, was recently approved by the FDA for treatment of cryopyrinassociated periodic syndromes. ${ }^{28}{ }^{29}$ Our data suggest that IL1 Trap has therapeutic potential for both the prophylaxis and treatment of acute gouty arthritis.

Acknowledgements: We thank Regeneron colleagues for advice and assistance.

Funding: RAT received support from the VA Research Service. RL-B received support from NIH AR1067966.

Competing interests: Regeneron Pharmaceuticals employed most authors, funded the work and holds a proprietary interest in the IL1 Trap reagent.

Provenance and peer review: Not commissioned; externally peer reviewed.

\section{REFERENCES}

1. Nuki G, Simkin PA. A concise history of gout and hyperuricemia and their treatment Arthritis Res Ther 2006;8(Suppl 1):S1.

2. Cronstein BN, Terkeltaub R. The inflammatory process of gout and its treatment. Arthritis Res Ther 2006;8(Suppl 1):S3.

3. Dalbeth $\mathbf{N}$, Haskard DO. Mechanisms of inflammation in gout. Rheumatology (Oxford) 2005;44:1090-6.

4. Masseoud D, Rott K, Liu-Bryan R, et al. Overview of hyperuricaemia and gout. Curr Pharm Des 2005;11:4117-24.

5. Terkeltaub R. Gout in 2006: the perfect storm. Bull NYU Hosp Jt Dis 2006;64:82-6.

6. Malawista SE, Duff GW, Atkins E, et al. Crystal-induced endogenous pyrogen production. A further look at gouty inflammation. Arthritis Rheum 1985;28:1039-46.

7. Di Giovine FS, Malawista SE, Nuki G, et al. Interleukin 1 (IL 1) as a mediator of crystal arthritis. Stimulation of T cell and synovial fibroblast mitogenesis by urate crystal-induced IL 1. J Immunol 1987;138:3213-8.

8. Agostini L, Martinon F, Burns K, et al. NALP3 forms an IL-1beta-processing inflammasome with increased activity in Muckle-Wells autoinflammatory disorder. Immunity 2004:20:319-25.

9. Martinon F, Petrilli V, Mayor A, et al. Gout-associated uric acid crystals activate the NALP3 inflammasome. Nature 2006;440:237-41.

10. Martinon F, Tschopp J. Inflammatory caspases and inflammasomes: master switches of inflammation. Cell Death Differ 2007;14:10-22.

11. Chen CJ, Shi Y, Hearn A, et al. MyD88-dependent IL-1 receptor signaling is essential for gouty inflammation stimulated by monosodium urate crystals. J Clin Invest 2006;116:2262-71

12. So A, De Smedt T, Revaz S, et al. A pilot study of IL-1 inhibition by anakinra in acute gout. Arthritis Res Ther 2007;9:R28.

13. Liu-Bryan R, Scott P, Sydlaske A, et al. Innate immunity conferred by Toll-like receptors 2 and 4 and myeloid differentiation factor 88 expression is pivotal to monosodium urate monohydrate crystal-induced inflammation. Arthritis Rheum 2005:52:2936-46.

14. Economides AN, Carpenter LR, Rudge JS, et al. Cytokine traps: multi-component, high-affinity blockers of cytokine action. Nat Med 2003;9:47-52

15. Torres R, Croll SD, Vercollone $\mathrm{J}$, et al. Mice genetically deficient in neuromedin $U$ receptor 2, but not neuromedin $U$ receptor 1 , have impaired nociceptive responses. Pain 2007:130:267-78.

16. Edwards JC, Sedgwick AD, Willoughby DA. The formation of a structure with the features of synovial lining by subcutaneous injection of air: an in vivo tissue culture system. J Pathol 1981:134:147-56. 
17. Roseff $\mathbf{R}$, Wohlgethan JR, Sipe JD, et al. The acute phase response in gout. J Rheumatol 1987;14:974-7.

18. Getting SJ, Lam CW, Chen AS, et al. Melanocortin 3 receptors control crystalinduced inflammation. FASEB J 2006:20:2234-41.

19. Matsukawa A, Yoshimura T, Maeda T, et al. Analysis of the cytokine network among tumor necrosis factor alpha, interleukin-1beta, interleukin-8, and interleukinreceptor antagonist in monosodium urate crystal-induced rabbit arthritis. Lab Invest 1998;78:559-69.

20. Coderre TJ, Wall PD. Ankle joint urate arthritis (AJUA) in rats: an alternative animal model of arthritis to that produced by Freund's adjuvant. Pain 1987;28:379-93.

21. Coderre TJ, Wall PD. Ankle joint urate arthritis in rats provides a useful tool for the evaluation of analgesic and anti-arthritic agents. Pharmacol Biochem Behav 1988;29:461-6.

22. Asano N, Morita T, Watanabe Y. Micronucleus test with colchicine given by intraperitoneal injection and oral gavage. Mutat Res 1989:223:391-4.

23. Zweig MH, Maling HM, Webster ME. Inhibition of sodium urate-induced rat hindpaw edema by colchicine derivatives: correlation with antimitotic activity. J Pharmacol Exp Ther 1972;182:344-50.
24. Rovin BH, Tan LC. Role of protein kinase pathways in IL-1-induced chemoattractant expression by human mesangial cells. Kidney Int 1994;46:1059-68.

25. Harkness KA, Sussman JD, Davies-Jones GA, et al. Cytokine regulation of MCP-1 expression in brain and retinal microvascular endothelial cells. J Neuroimmuno 2003;142(:1-9

26. Persson-Dajotoy T, Andersson P, Bjartell A, et al. Expression and production of the CXC chemokine growth-related oncogene-alpha by human eosinophils. J Immunol 2003;170:5309-16.

27. Ren K, Torres R. Role of Interleukin-1 beta during pain and inflammation. Brain Res Rev 2009;60:57-64.

28. Schafers $\mathbf{M}$, Sorkin L. Effect of cytokines on neuronal excitability. Neurosci Lett 2008;437:188-93.

29. Goldbach-Mansky R, Shroff SD, Wilson M, et al. A pilot study to evaluate the safety and efficacy of the long-acting interleukin-1 inhibitor rilonacept (interleukin-1 Trap) in patients with familial cold autoinflammatory syndrome. Arthritis Rheum 2008;58:2432-42.

30. Hoffman HM, Throne ML, Amar NJ, et al. Efficacy and safety of rilonacept (interleukin-1 Trap) in patients with cryopyrin-associated periodic syndromes: results from two sequential placebo-controlled studies. Arthritis Rheum 2008;58:2443-52.

\section{Take advantage of BMJ Journals' remarkable catalogue of titles with Related Collections}

No busy professional has time to browse through all pertinent journals to find relevant articles, but with Related Collections you no longer have to. Follow the "Related Collections" link from any article and use the "Show Collections from other Journals" to expand your search across all BMJ Journals. Or simply follow the "Browse by topic" link on the home page. By setting up your own collections and receiving email alerts every time an article is added to your chosen area, you can build up your own significant body of knowledge. 